\title{
Time resolved digital holography applied to droplets fragmentation by shockwave
}

\author{
Zacaria Essaïdi, Pierre Lauret, Pierre R.L. Slangen \\ IMT Mines Ales, Universite de Montpellier, LGEI-ISR, 6 avenue de Clavieres 30319 Ales Cedex France
}

\begin{abstract}
Droplets atomization by shockwave can occur in different issues commonly encountered in the industry such as leak, tank leakage or triple aggression (high speed impact, rupture and surrounded secondary explosion) of tanks. For the last case, shockwave can interact with liquid jets of drops and propagates the liquid far away from the container zone. Very fine secondary droplets can be produced in the worst case of atomization. These small particles can generate secondary effects like explosion in case of petrol derivatives in fire or toxic effects in case of direct breathing.

High speed imaging is well suited to study transient phenomenon like explosions and shockwave. A dedicated shockwave generator has been designed to cope with interferometric measurement on holographic bench. This demonstrator is made of thick plastic tubes. The high pressure chamber is isolated from the guiding tube by domestic aluminum foils, the thickness and number of which drive the pressure rupture. Previous works have been carried on by time resolved shadowgraphy to characterize generated shockwave at the guiding tube outlet.

This paper deals with time resolved digital holography to perform higher accuracy measurements and of course to reach 3D reconstruction of the whole phenomenon. Lensless in-line digital holography is carried on to improve the stability of the holographic set-up. Different Phantom high speed cameras have been tested as recording sensors, following pixel pitch, pixel size and of course the maximal throughput, from 7kfps (frame per second) up to 26kfps at full 1Mpixel resolution.
\end{abstract}

Different regimes of droplet trains and droplet sizes have been tested. This has also been carried on for different liquids to show the effect of the physico-chemical properties of the liquid subjected to shockwave.

Keywords: shockwave, droplets, fragmentation, digital holography; high speed imaging, laser, speckle

\section{INTRODUCTION}

Aerodynamic fragmentation is a physical phenomenon involving the forces of inertia of the flow and the forces of cohesion of drops. Thus, when aerodynamic forces exceed the forces of surface tension, droplet ("mother") is deformed and breaks leading to smaller secondary drops ("daughters"). This mechanism is well documented and the associated Weber numbers for this study are in perfect agreement with the literature ${ }^{1,2}$. The aim of this paper is to understand this phenomenon, as it can occur in scenarios of risks ${ }^{3,4}$ or for the mitigation of the effects of the explosions by water curtains. In order to analyze at the laboratory scale this process of fragmentation and the girls drops, shock tube have been designed coupled with a time resolved digital holography set-up. High speed imaging acquisition rates up to $24 \mathrm{kHz}$ have been reached to catch the particles after atomization. Both main principles and basic rules of high speed imaging have been extensively discussed in previous paper of the same research group ${ }^{5}$. In line digital holography ${ }^{6}$ will be performed as it is robust to vibrations and allows high resolution reconstructions of small objects. The shock tube and the generated shockwave will be described as well as the camera performances used to grab the images sequence in high speed. This set-up is applied to different liquids e.g. ethanol, water, and water mixed with different PEG (PolyEthyleneGlycol) concentration to obtain a large panel of Weber number. The drop dispenser rate is adjusted to get a gentle train of drops during the shock. Shock generation is trigging the rolling buffer camera. Raw images are then postprocessed by angular spectrum back propagation in the object plane to reconstruct the shock and the droplets amplitude

*pierre.slangen@mines-ales.fr 
image before and after the shock. Future developments will be discussed such as double slanted in-line applications ${ }^{7}$ and Mach-Zender holographic interferometer to compute the phase ${ }^{8}$.

\section{METHODOLOGY}

In this section, the main properties of droplets fragmentation, the shock tube generator and the digital holography processing will be described.

\subsection{Droplets fragmentation}

Some dimensionless numbers in fluid mechanics ${ }^{9}$ are governing the fragmentation process of liquid drops propagating in the surrounding fluid medium. Among these, we can get:

Weber number, $\boldsymbol{W} \boldsymbol{e}=\boldsymbol{\rho} \boldsymbol{V}^{2} \boldsymbol{L}_{\boldsymbol{C}} / \boldsymbol{\sigma}$, is used to characterize the flow of fluids to the interface of a multiphase system. It is defined as the ratio of the aerodynamic forces (forces of inertia) and cohesion forces (surface tension), with $V$, the speed of the fluid $\left[\mathrm{m} . \mathrm{s}^{-1}\right], L_{c}$, the characteristic length $[\mathrm{m}], \rho$ the density $\left[\mathrm{kg} \cdot \mathrm{m}^{-3}\right]$ and $\sigma$ the surface tension $\left[\mathrm{N} \cdot \mathrm{m}^{-1}\right]$.

Reynolds number, $\boldsymbol{R} \boldsymbol{e}=\boldsymbol{\rho} \boldsymbol{V} \boldsymbol{L}_{\boldsymbol{c}} / \boldsymbol{\mu}$, where $\mu$ is the dynamic viscosity [Pa.s], describes the flow regime (laminar, turbulent, transitional). It is the ratio of the forces of inertia and viscous forces.

Ohnesorge number, $\boldsymbol{O} \boldsymbol{h}=\boldsymbol{\mu}\left(\boldsymbol{\rho} \boldsymbol{L}_{C} \boldsymbol{\sigma}\right)^{-1 / 2}$, describes the drops deformations. It is the ratio of the forces of viscosity and the square root of the product of the cohesion and inertia forces.

Pilch and Erdman ${ }^{1}$ extensively give the modes of drop fragmentation with respect to these numbers. It is resumed by Figure 1.

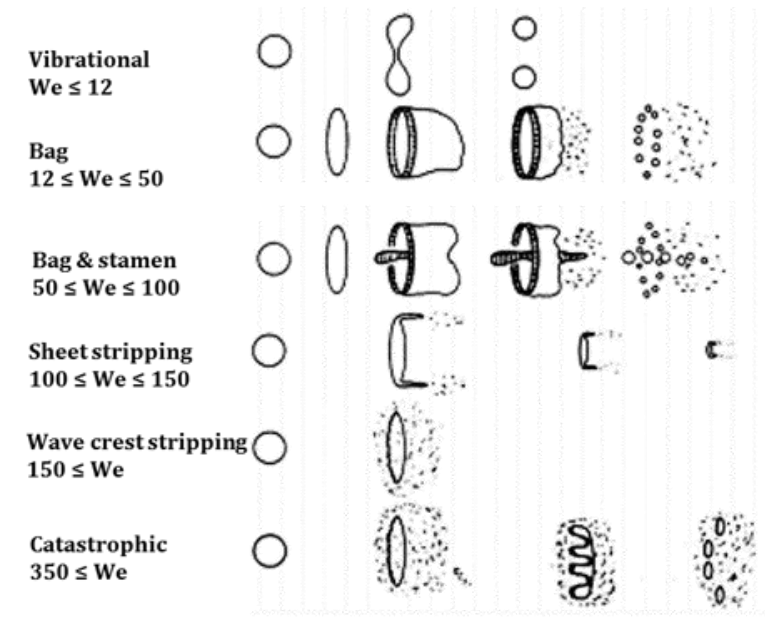

Figure 1. Main regimes of fragmentation governed by We number

This study will mainly focus on conditions governed by Weber numbers from wave crest stripping to catastrophic modes. Therefore, drops of ethanol, isopropanol, pure water, and mix of water $+\mathrm{X} \%$ of PEG (PolyEthyleneGlycol, $\mathrm{X}$ from $0 \%$ to $100 \%$ ) will be generated from the drop dispenser to cope with these We numbers.

\subsection{Shockwave generation}

Shockwave generator has been designed to fit with the vibrations insulation optical bench. This enables to apply Time resolved digital holographic interferometry (TRDHI) for measuring the interaction of shockwave and droplets. This light open-ended shock tube is made of plastic pipe with $63 \mathrm{~mm}$ inner diameter, $3 \mathrm{~mm}$ thick, from standard high pressure plumbery (PVC). The high pressure chamber $(700 \mathrm{~mm}$ long) is separated for the outlet pipe (300 $\mathrm{mm}$ long) by reinforced $20 \mu \mathrm{m}$ thick aluminum sheets (for food). The number of sheet is driving the rupture pressure. For 4 sheets, the overpressure is about $100 \mathrm{mbar}$ at $17 \mathrm{~cm}$ from the exit, measured by PCB high speed pressure sensor. From the optical measurement, speed of the shockwave is around $380 \mathrm{~m} / \mathrm{s}$. This has been previously measured in high speed shadowgraphy (1Mpixel, 25kfps, $1 \mu$ s exposure), by Z-shaped shadowgraphy ${ }^{10}$ system using point source coupled with parabolic first surface mirrors, $\mathrm{f} / 8,33 \mathrm{~cm}$ in diameter. Shadowgraphs and streak image (time versus distance graph along 
the shock tube axis) are presented: the shock is followed by high speed $(100 \mathrm{~m} / \mathrm{s})$ blow and low speed $(30 \mathrm{~m} / \mathrm{s})$ vortex ring (Figure 2). The flow propagation is from left to right by convention.
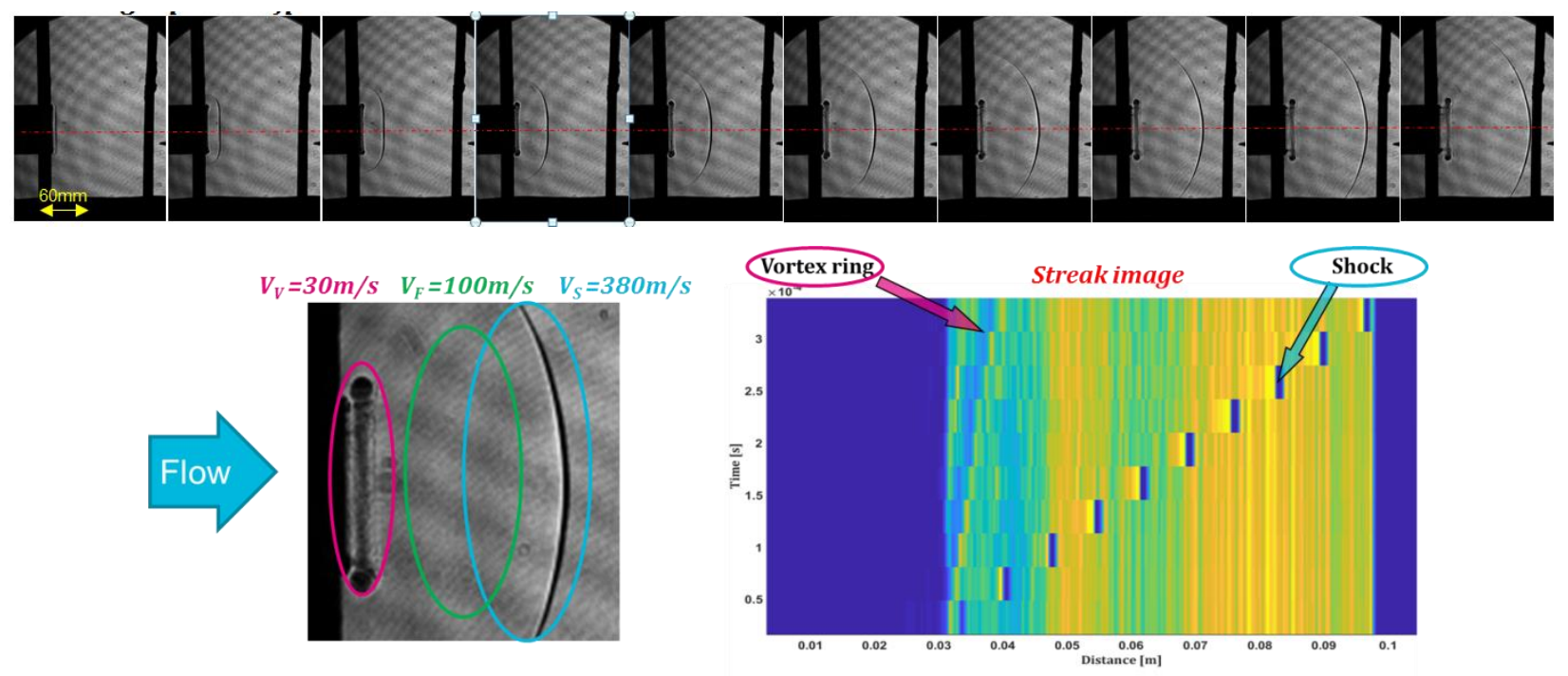

Figure 2. Shadowgraphs of shockwave outlet $(25 \mathrm{kfps}, 40 \mu \mathrm{s}$ between successive images) and streak image

\subsection{Time resolved digital holography}

Digital holography ${ }^{11,12}$ is very powerful in metrology and can now be applied in high speed acquisition (or time resolved) thanks to sensitive sensors enabling short exposure time ( $1 \mu \mathrm{s}$ or less), high throughput (26Mpix/s and more) with rather small pixel size (from 10 to $30 \mu \mathrm{m}$ ). The size of the pixel sensor directly acts on the smallest detail to be reconstructed $^{13,14}$. In-line or Gabor set-up is used to be less sensitive to external vibrations e.g. generated by the shocktube (Figure 3).

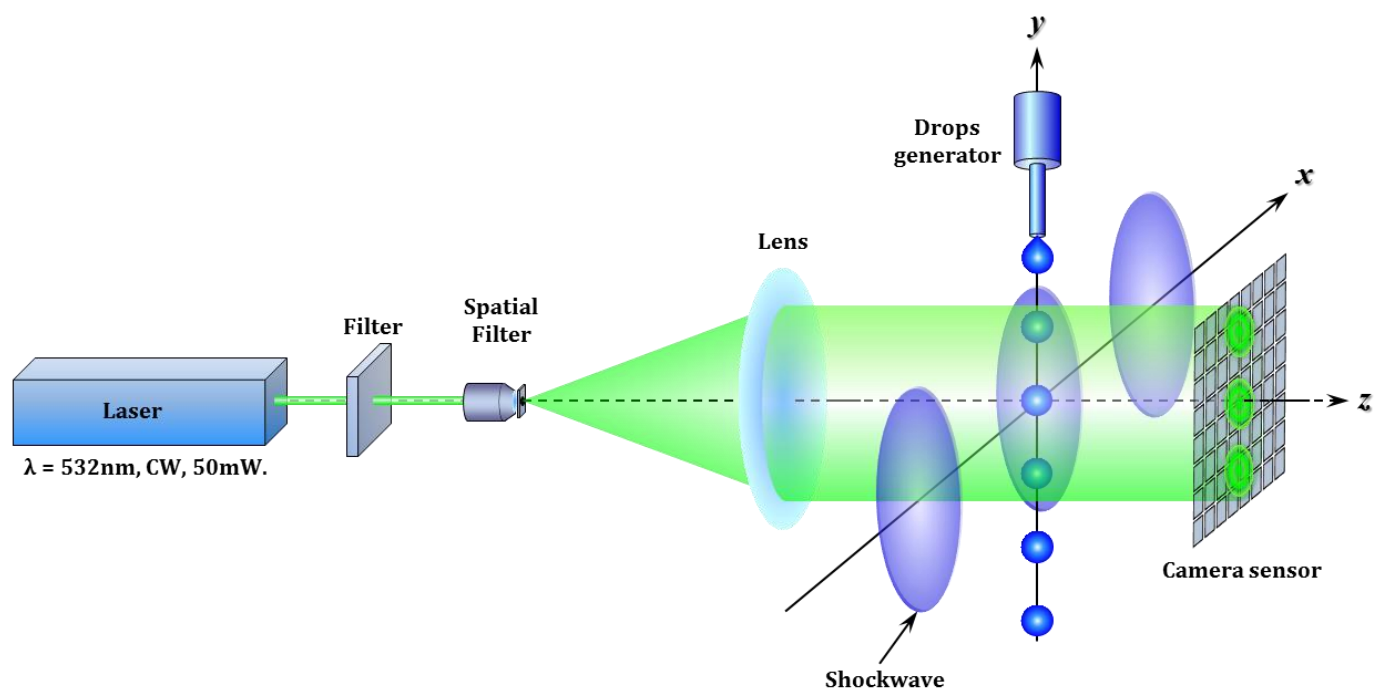

Figure 3. In-line digital holography set-up

The laser beam is spatially filtered and collimated by the lens $(\mathrm{f}=+500 \mathrm{~mm})$. High speed image sensor is placed at about $30 \mathrm{~cm}$ of the shocktube axis to avoid direct damage to the lensless camera. This distance enables safe camera and rather good reconstruction resolution after recording. Two different cameras have been used: Vision Research Phantom V710

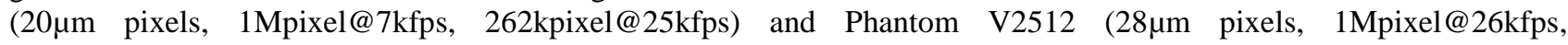
630kpixel@38kfps). Regarding constant throughput, the frame rate can be enhanced by reduced sensor resolution. 
Droplets are falling from the needle tip of remote-controlled syringe, the diameter of which can be adjusted to achieve different drop sizes. It is aligned along the optical axis of the holographic set-up in front of the shock generator as shown on the lab picture (Figure 4).

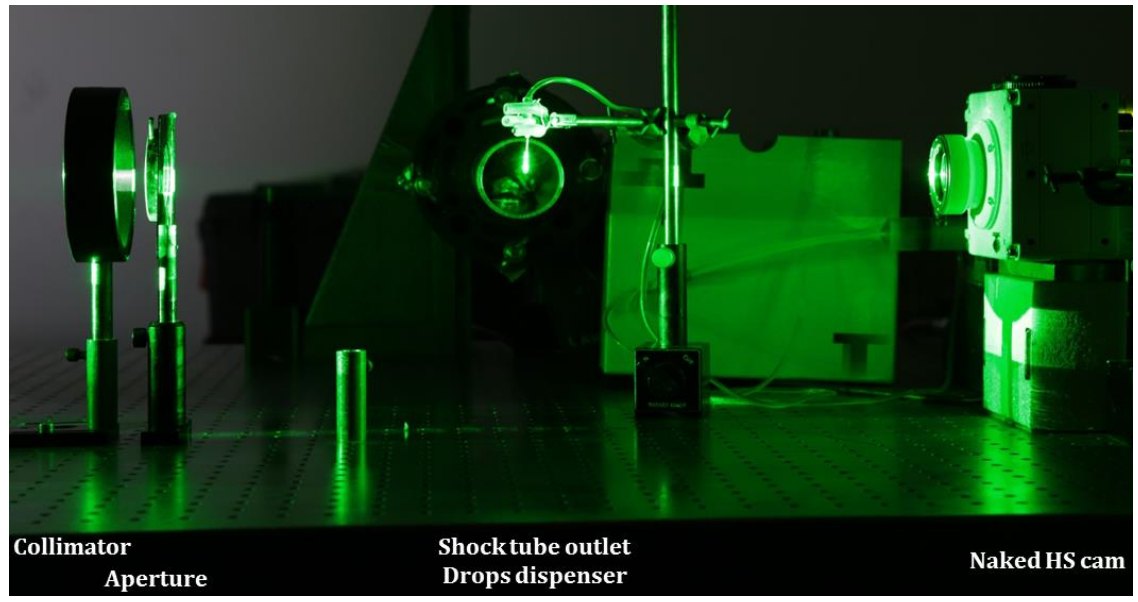

Figure 4. Time resolved in-line digital holography set-up

The recorded holograms are post processed by considering the scalar diffraction theory. The real image is back propagated to the reconstruction plane located at distance $d_{0}$ by means of propagator operator. In this application, propagation is achieved using the angular spectrum propagation method ${ }^{15}$. The optimal reconstruction distance is reached at maximal local contrast, i.e. best focus on the mother droplet, for $d_{0}=333.4 \mathrm{~mm}$ (Figure 5).

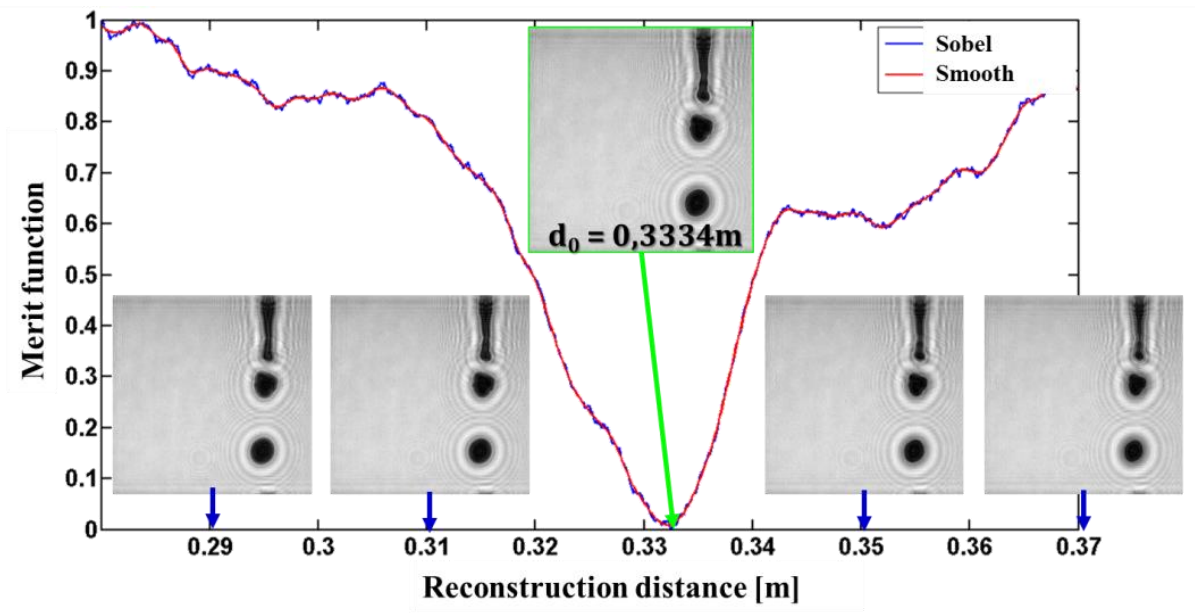

Figure 5. Automatic "best focus" reconstruction distance

The twin image presents in the reconstruction is not relevant as it can be suppressed by former image processing. Some authors even use digital modeling from the diffracted image to recover the object ${ }^{16}$ but this method cannot be applied here as a lot of interfering secondary droplets are generated after the shock. In this study, magnification is unitary $(\mathrm{M}=1)$, and so the minimal particle size is about 2 pixels wide i.e. $40 \mu \mathrm{m}$ for VEO710 and $56 \mu \mathrm{m}$ for V2512. Some authors have shown higher magnification to reach about $7.2 \mu \mathrm{m}$ sensitivity, while considering $3 \mathrm{x}$ the effective pixel size of $2.4 \mu \mathrm{m}^{7}$.

\section{RESULTS AND DISCUSSION}

As above-mentioned, this study is mainly applied on catastrophic and stripping fragmentation modes. 
These are the most dangerous modes as droplets are rapidly dispersed in very fine secondary droplets forming clouds. These clouds can be submitted to wind, or secondary air flow, and so reach population or responder in case of industrial accident. Toxic products can then be inhaled, and flammable products can easily explode.

For the liquids under study, water+100\% PEG is the more viscous and ethanol the more volatile.

Properties of drops, flow speed, and dimensionless numbers are summarized by Table 1.

Table 1: Physico-chemical properties of involved liquids and shock tube flow properties

\begin{tabular}{|c|c|c|c|}
\hline & Liquids & & \\
\hline \multirow{2}{*}{ Characteristics } & PEG & Water & Ethanol \\
\hline & $\mathrm{H}\left(\mathrm{OCH}_{2} \mathrm{CH}_{2}\right)_{\mathrm{n}} \mathrm{OH}$ & $\mathrm{H}_{2} \mathrm{O}$ & $\mathrm{C}_{2} \mathrm{H}_{6} \mathrm{O}$ \\
\hline Drops diameter $D[\mathrm{~m}]$ & $1.7 \times 10^{-3}$ & $1.2 \times 10^{-3}$ & $0.95 \times 10^{-3}$ \\
\hline Drop deformation time $[\mu \mathrm{s}]$ & 708.22 & 499.92 & 374.94 \\
\hline Flow velocity $v_{2}\left[\mathrm{~m} \cdot \mathrm{s}^{-1}\right]$ & 100 & 100 & 100 \\
\hline Weber number We & 451 & 195 & 507 \\
\hline Reynolds number Re & $1,08 \times 10^{4}$ & $7,65 \times 10^{3}$ & $6,06 \times 10^{3}$ \\
\hline Ohnesorge number Oh & $3,26 \times 10^{-1}$ & $3,38 \times 10^{-3}$ & $9,32 \times 10^{-3}$ \\
\hline
\end{tabular}

For each liquid, the experiment is conducted 5 times for reliability purpose. Syringe dispenser is actuated to get the droplets train. Then the high pressure chamber is inflated until disk rupture. High speed camera is free running in rolling buffer memory (FIFO, first in first out, mode). It is triggered by the disk rupture. On board camera memory images sequence (up to $92 \mathrm{~GB}$ ) is then transferred to computer for saving and post processing. These experiments are generating a huge amount of data storage, as 12 bits raw images (or uncompressed TIFF) must be kept through whole processing to ensure high quality (spatial and temporal) resolutions.

\subsection{Shockwave diffraction}

Thanks to holographic time resolved measurement and high spatial resolution coupled with good contrast, it is even possible to show the diffraction of the shockwave by the falling drops, before atomization.
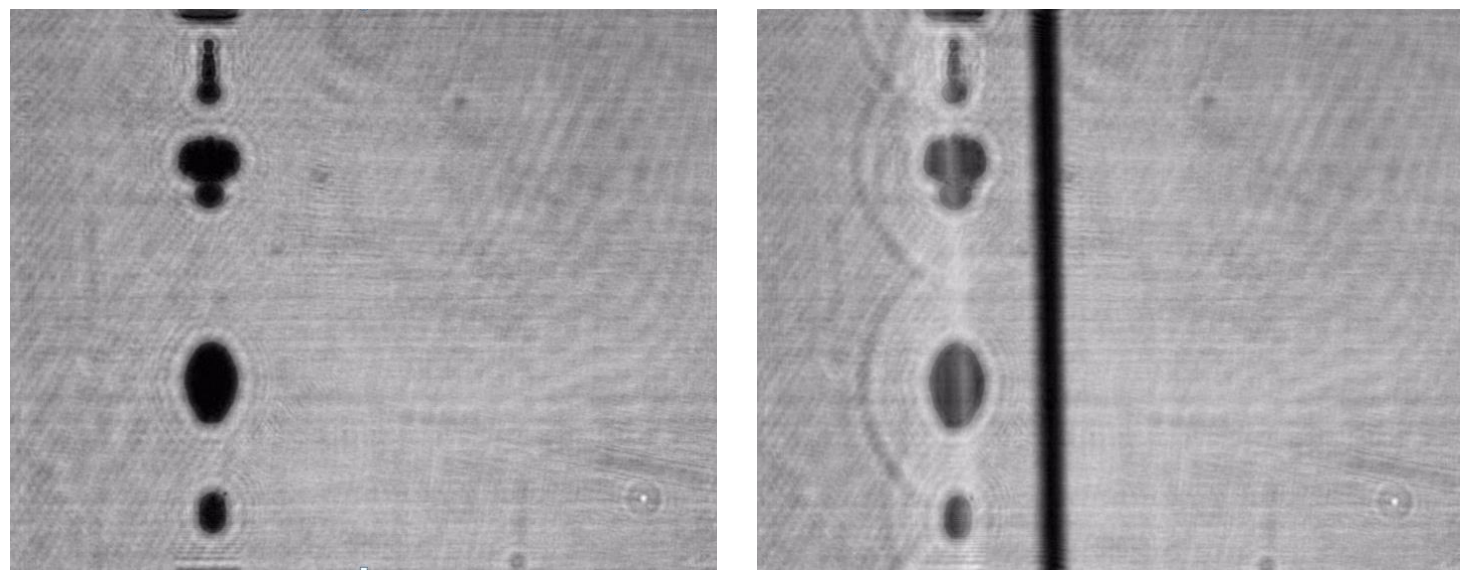

Figure 6. Water drops ( $2 \mathrm{~mm})$ before shock and diffracted shock, $\mathrm{M}=1(28 \mu \mathrm{m} / \mathrm{pixel}), 38 \mathrm{kfps}, 1 \mu \mathrm{s}$, Phantom V2512

Although the weak shock with the small shock tube, it is well defined on Figure 6 where the shock is generated from $4 \mathrm{bar}$ disk rupture. The shockwave propagates from left to right, by convention. 


\subsection{Droplets fragmentation}

The different liquids have been tested in the conditions reported in Tab.1. The holographic temporal evolution from the ethanol to $100 \%$ PEG is presented by Figure 7 . The ethanol is the less viscous while 100\%PEG is the more viscous. Increasing concentration from pure water to $100 \%$ PEG have been carried on but not shown here for simplicity.

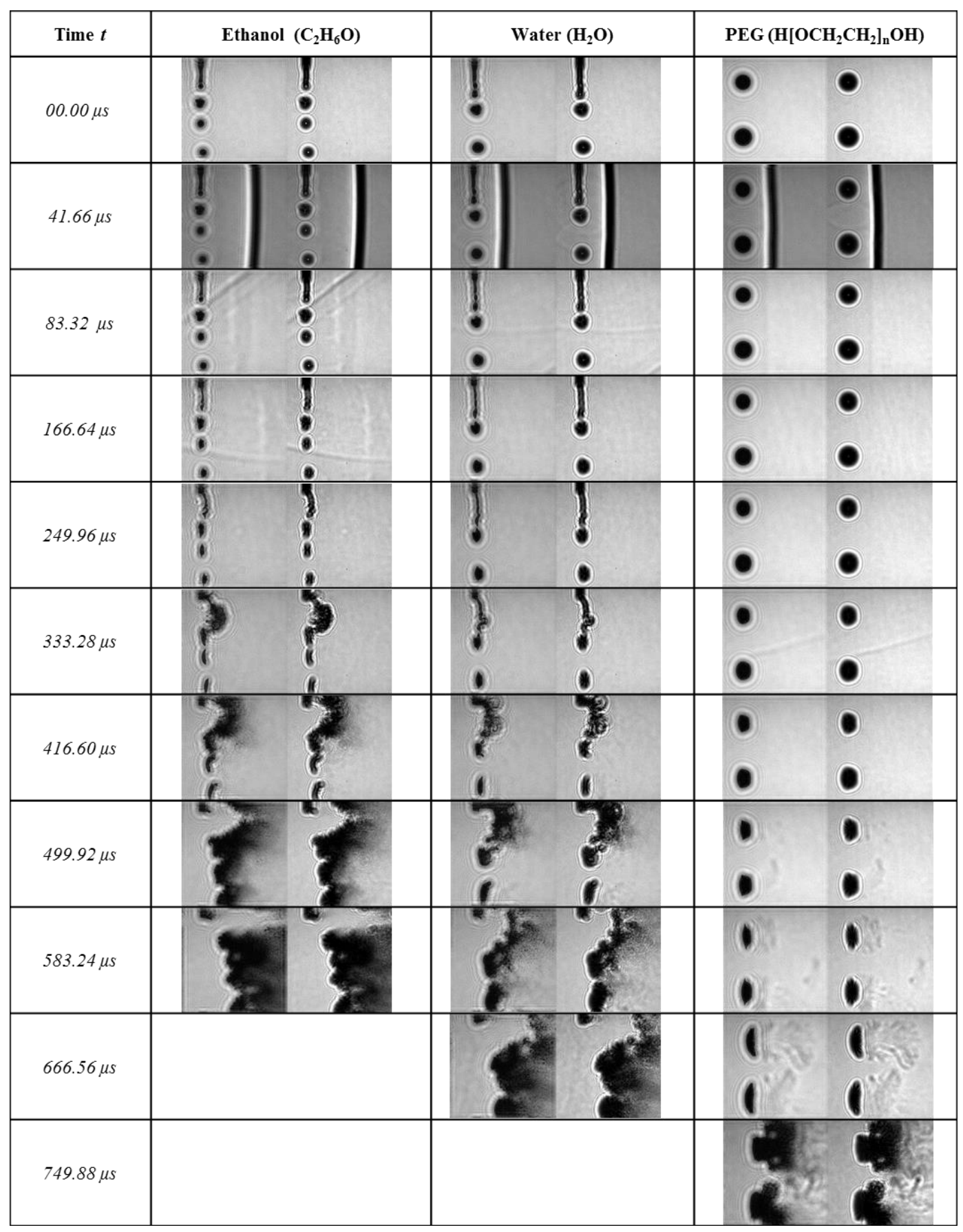

Figure 7. Time resolved evolution for different liquids (from less to more viscous).

The main advantage of holography compared to direct shadowgraphy is the ability to reconstruct the shape or the droplet distribution in 3D (i.e. 3D information recorded on 2D support). This is achieved through "best focus picking" (Figure 5) 
for different regions of interest in the reconstructed hologram (Figure 8). It is then possible to reconstruct the droplet (shape and location) and even wires or trails after fragmentation.

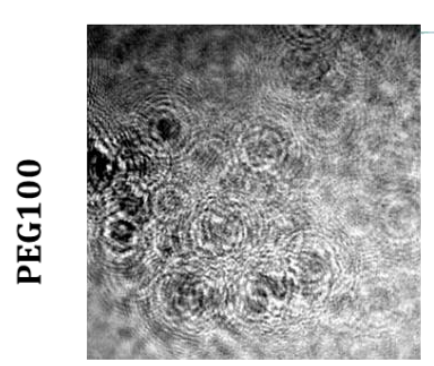

Hologram

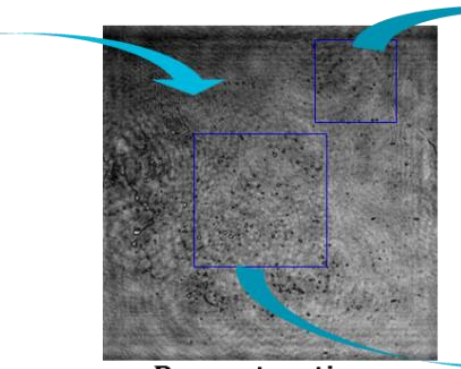

Reconstruction

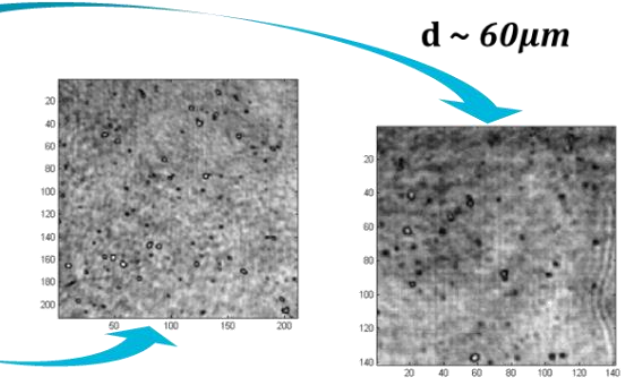

Figure 8. Holographic reconstruction for 100\% PEG in different regions of interest.

Figure 9 presents the evolution of the droplet profile after the shock and the main flow. Transfer of matter from the inward to the outward side of the drops is clearly reported for each liquid. This confirms theoretical works from Liu ${ }^{17}$.
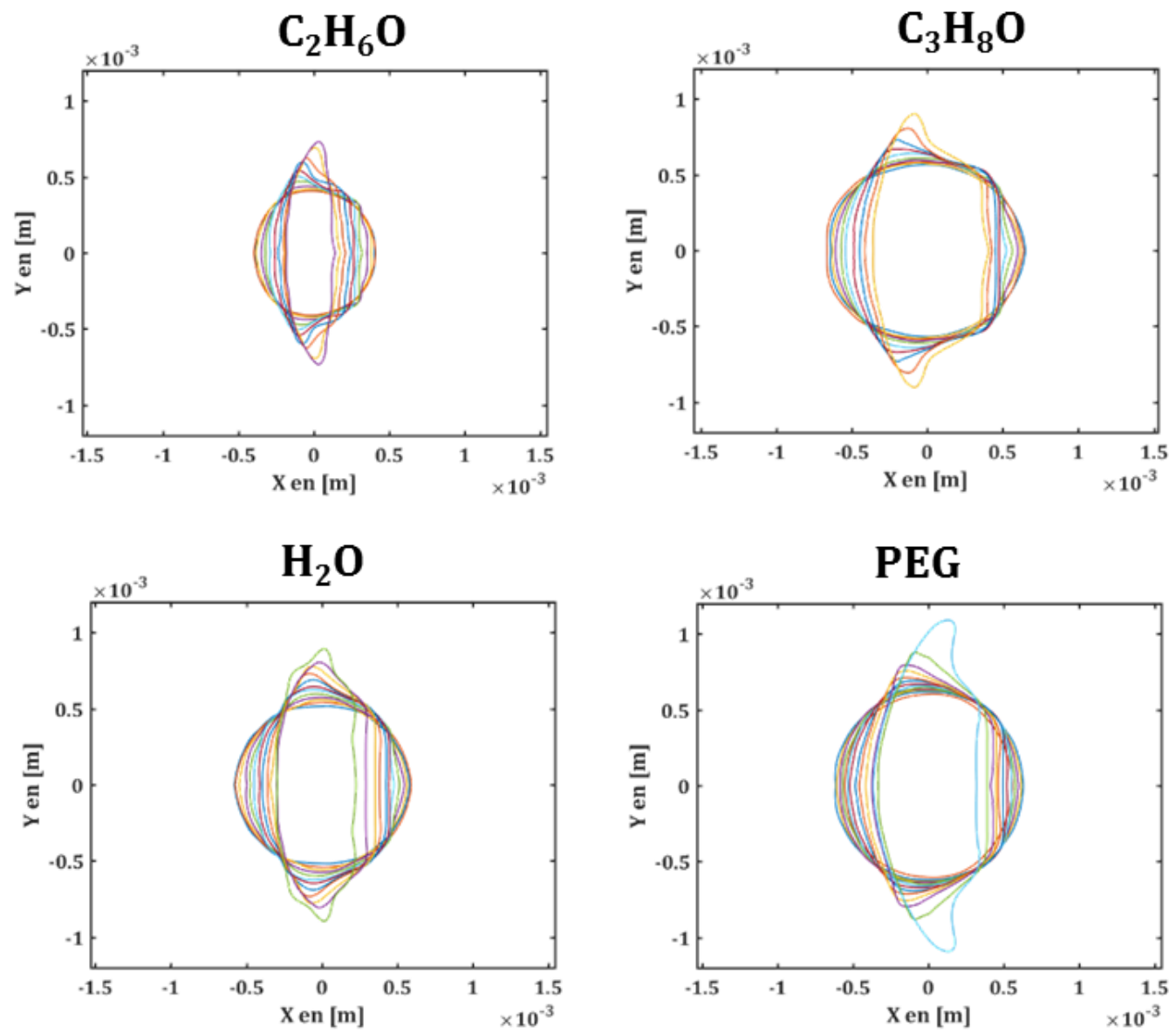

Figure 9. Time resolved evolution for different liquids (from less to more viscous).

As holographic recording lasts during the whole memory buffer, it is even possible to catch small droplets interacting with the "second" flow produced by the reflexion of the initial shock on the closed side of the shock tube. This weakest flow can then produce fragmentation for smaller We number, producing bag breakup for example. It is clearly visible even if the contrast is very poor due to hot gases and the huge amount of secondary small particles (Figure 10). 

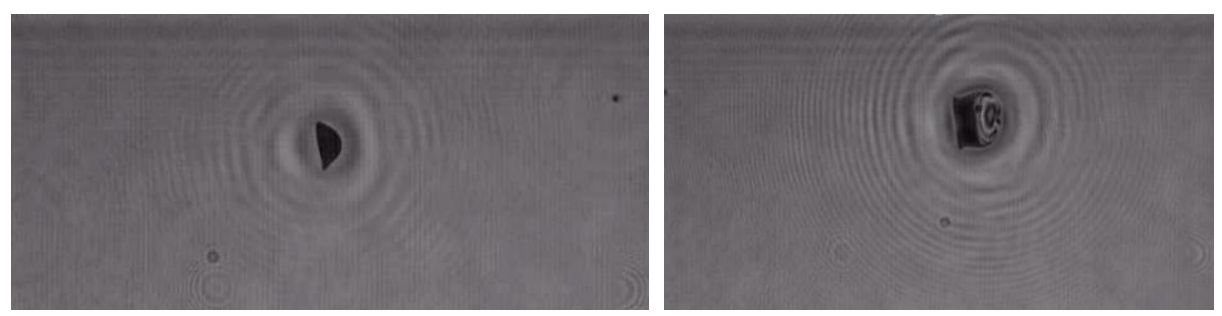

Figure 10. Example of bag breakup by reflected flow for secondary PEG drop.

\section{CONCLUSION}

This study focus on the application of time resolved digital holography on droplets fragmentation by shockwave. The main advantage of holography is the possibility to reconstruct the droplets (mother and daughters) in $3 \mathrm{D}$, i.e. in shape (using multiple views) and location. Therefore the focus picking reconstruction is carried on by local best contrast tracking. The application has been demonstrated for different liquids showing low and high viscosity. The results enable to track the particles in $3 \mathrm{D}$ and to generate automatic reports showing the size distribution of the droplets. This will be soon extended to $3 \mathrm{D}$ reports as shown by Gao et $\mathrm{al}^{18}$. The shockwave tube has been designed to cope with the vibration insulated bench, but the next step will be to apply in-line time resolved holography on long, high pressure shock tube. Some authors ${ }^{8}$ also report the possibility to Mach-Zender configuration in presence of shockwave, so as to be able to compute the phase of droplets. This configuration will be tested in the next future, firstly on the small tube and afterwards on the "long range" tube.

\section{REFERENCES}

[1] Pilch, M. and Erdman, C. A., "Use of breakup time data and velocity history data to predict the maximum size of stable fragments for acceleration-induced breakup of a liquid drop,” Int. J. Multiph. Flow 13(6), 741-757 (1987).

[2] Guildenbecher, D. R., López-Rivera, A. C., Sojka, A. P. E., López-Rivera, C., Sojka, P. E. and Laboratories, M. J. Z., "Secondary atomization," Exp Fluids 46(3), 371-402 (2009).

[3] Heymes, F., Aprin, L., Slangen, P., Lapébie, E., Osmont, A. and Dusserre, G., "On the Effects of a Triple Aggression (Fragment, Blast, Fireball) on an LPG Storage,” Chem. Eng. Trans. 36, 355-360 (2014).

[4] Lauret, P., Heymes, F., Slangen, P., Aprin, L. and Lecysyn, N., "Consequences of liquid jet breakup resulting from interaction with overpressure wave from domino effect," Chem. Eng. Trans. 53, 73-78 (2016).

[5] Slangen, P. R., Essaidi, Z., Chanut, C., Lauret, P., Heymes, F. and Aprin, L., "Recent developments in high speed imaging and applications in speckle light," Proc. SPIE SPECKLE 2018 VII Int. Conf. Speckle Metrol.(September 2018), 36, SPIE-INT SOC OPTICAL ENGINEERING, Janów Podlaski (2018).

[6] Poittevin, J., Picart, P., Faure, C., Gautier, F. and Pézerat, C., "Multi-pont vibrometer based on high-speed digital in-line holography," Appl. Opt. 54(11), 3185-3196 (2015).

[7] Guildenbecher, D. R., Gao, J., Chen, J. and Sojka, P. E., "Characterization of drop aerodynamic fragmentation in the bag and sheet-thinning regimes by crossed-beam, two-view, digital in-line holography," Int. J. Multiph. Flow 94, 107-122 (2017).

[8] Olchewsky, F., Essaïdi, Z., Desse, J.-M. and Champagnat, F., "3D reconstructions of jets by multidirectional digital holographic tomography," 18th Int. Symp. Flow Vis. 3D, Zurich, Switzerland (2018).

[9] Tropea, C., Yarin, A. L. and Foss, J. F., [Springer Handbook of Experimental Fluid Mechanics], Springer Science+Business Media (2007).

[10] Settles, G. S. and Hargather, M. J., "A review of recent developments in schlieren and shadowgraph techniques," Meas. Sci. Technol. 28(4), 042001 (2017).

[11] Kreis, T., "Digital holography for metrologic applications," Interferom. SPECKLE Light THEORY Appl., Jacquot, P and Fournier, JM, Ed., 205-212, SPRINGER-VERLAG BERLIN, HEIDELBERGER PLATZ 3, D14197 BERLIN, GERMANY (2000).

[12] Picart, P. and Li, J., [Digital holography], Hoboken, NJ USA (2012).

[13] Picart, P. and Leval, J., "General theoretical formulation of image formation in digital Fresnel holography," J. Opt. Soc. Am. A-OPTICS IMAGE Sci. Vis. 25(7), 1744-1761 (2008).

[14] Karray, M., Slangen, P. and Picart, P., "Comparison between Digital Fresnel Holography and Digital Image- 
Plane Holography: The Role of the Imaging Aperture,” Exp. Mech. 52(9), 1275-1286 (2012).

[15] Schnars, U., Falldorf, C., Watson, J. and Jüptner, W., [Digital Holography and Wavefront Sensing], SpringerVerlag, Berlin/Heidelberg (2014).

[16] Berdeu, A., Momey, F., Laperrousaz, B., Bordy, T., Gidrol, X., Dinten, J.-M., Picollet-D’hahan, N. and Allier, C., "Comparative study of fully three-dimensional reconstruction algorithms for lens-free microscopy," Appl. Opt. 56(13), 3939-3951 (2017).

[17] Liu, N., Wang, Z., Sun, M., Wang, H. and Wang, B., "Numerical simulation of liquid droplet breakup in supersonic flows," Acta Astronaut. 145, 116-130 (2018).

[18] Gao, J., Guildenbecher, D. R., Reu, P. L., Kulkarni, V., Sojka, P. E. and Chen, J., "Quantitative, threedimensional diagnostics of multiphase drop fragmentation via digital in-line holography.," Opt. Lett. 38(11), 1893-1895 (2013). 